\title{
Medicina conductual: trabajo en la clínica del dolor del Hospital Juárez de México
}

\section{Behavioral medicine: work in the pain clinic of the Hospital Juárez de México}

\author{
Guadalupe A. Hernández-Gálvez ${ }^{1 *}$, Ana L. Becerra-Gálvez², Diana Salazar-Díaz ${ }^{1}$ y \\ Patricia Hernández-Solís ${ }^{3}$ \\ ${ }^{1}$ Programa de Maestría y Doctorado en Psicología, Universidad Nacional Autónoma de México; ${ }^{2}$ Tutoría y Supervisión académica, Residencia en \\ Medicina Conductual, Universidad Nacional Autónoma de México; ${ }^{3}$ Clínica del Dolor y Cuidados Paliativos, Hospital Juárez de México. Ciudad \\ de México, México
}

\section{Resumen}

En el caso de los padecimientos crónicos que generan dolor, el especialista en medicina conductual trabaja de manera colaborativa con el personal de salud y coadyuva en el control del dolor diseñando intervenciones eficaces y ajustadas a las características, tipo de dolor y necesidades del paciente, para favorecer su adaptación al padecimiento doloroso y a las complicaciones propias de la etapa paliativa. La Clínica del Dolor y Cuidados Paliativos del Hospital Juárez de México cuenta con profesionales de la salud (médicos, enfermeros, psicólogos) cuyas líneas de acción están encaminadas a dar atención de calidad tanto al paciente como al cuidador y, así, de manera conjunta, mejorar su calidad de vida. El objetivo de este documento es describir la labor del médico conductual como parte de las actividades del equipo multidisciplinario para la valoración e intervención de los pacientes con dolor y/o en cuidados paliativos del Hospital Juárez de México.

Palabras clave: Medicina conductual. Algología. Enfermería. Manejo multidisciplinario. Dolor. Cuidados Paliativos.

\begin{abstract}
In chronic illness that generate pain, behavioral medicine experts work in conjunction with health personnel and assist in pain control by designing effective interventions adjusted to the characteristics, type of pain and needs of the patient to favor his adaptation to the painful condition and the complications associated in each of the palliative stages. The Pain and Palliative Care Clinic of Hospital Juárez de México has health professionals (doctors, nurses, psychologists) whose lines of action are targeted at providing quality care to both the patient and the caregiver and thus jointly improve their quality of life. The aim of this document is to describe the work of the behavioral medicine specialist as part of the activities of the multidisciplinary team for the assessment and intervention of patients with pain and/or palliative care at the Hospital Juarez de Mexico.
\end{abstract}

Key words: Behavioral Medicine. Algology. Nursing. Multidisciplinary Management. Pain. Palliative Care.

Correspondencia:

*Guadalupe A. Hernández-Gálvez

E-mail: arelehernandez@gmail.com licencia CC BY-NC-ND (http://creativecommons.org/licenses/by-nc-nd/4.0/).
Disponible en internet: 10-03-2020 Rev Hosp Jua Mex. 2020;87(1):43-50

www. revistahospitaljuarez.com 


\section{Introducción}

La Asociación Internacional para el Estudio del Dolor, en $2009^{1}$, define el dolor como «una experiencia sensorial desagradable asociada con una lesión hística real o potencial o que se describe como ocasionada por dicha lesión o cuya presencia es revelada por manifestaciones visibles $y / 0$ audibles de la conducta». El dolor desde esta perspectiva produce una serie de afectaciones de carácter físico y psicológico en el paciente $\mathrm{y}$, por ende, su calidad de vida se deteriora significativamente.

A pesar de la prevalencia de este tipo de trastornos, no se dispone de información suficiente para determinar la cantidad de personas con dolor crónico a nivel mundial. En el caso de México, los médicos de primer contacto reportan que hasta un $22 \%$ de sus consultas son pacientes que refieren dolor y 83 millones de adultos refieren que el dolor afecta algunas de sus actividades cotidianas ${ }^{2}$. Para cubrir esta demanda, desde 1953 se han constituido clínicas del dolor, que ayudan al manejo de este síntoma en los pacientes. La misión de estos servicios es otorgar diagnóstico y tratamientos médicos para el control del dolor y otros síntomas característicos de la etapa paliativa, por lo tanto, incluyen apoyo de especialistas como: algólogos, psicólogos, trabajadores sociales y personal de enfermería. De manera complementaria, se puede incluir el trabajo de otros especialistas como: médicos en rehabilitación, apoyo nutricio y soporte espiritual.

\section{Clínica del Dolor y Cuidados Paliativos del Hospital Juárez de México}

El incremento de los pacientes con dolor agudo y crónico que se atienden en el Hospital Juárez de México dio lugar en 2011 a la creación de la Clínica del Dolor y Cuidados Paliativos (CdDyCP) $)^{3}$, y a partir del 2012 fue aceptada como sede para la formación de médicos algólogos con aval de la Universidad Nacional Autónoma de México. En 2013 se integraron al equipo multidisciplinario residentes psicólogos del Programa de Maestría en Psicología con Residencia en Medicina Conductual de la Facultad de Estudios Superiores Iztacala de la Universidad Nacional Autónoma de México. Finalmente, en 2014 se implementó la asistencia médica y psicológica vía telefónica tanto a pacientes como a familiares ${ }^{4}$. Con la mira de proporcionar atención multidisciplinaria a los usuarios de la CdDyCP se han definido y sistematizado las actividades de cada uno de los especialistas, las cuales se describen a continuación.
Personal médico. Los algólogos son los encargados de identificar las características del dolor y tratar a los pacientes considerando la semiología del padecimiento doloroso. Para ello, identifican la base del dolor (oncológico o no oncológico), su temporalidad (si se trata de un dolor agudo o crónico) y su manejo médico previo (si se trata de un dolor de difícil control) $)^{5}$.

Personal de enfermería. Se encarga de coadyuvar en actividades realizadas por el médico, tales como la evaluación del dolor y sus afectaciones en las actividades de la vida cotidiana, administración de analgésicos y reconocimiento temprano de los efectos adversos y, en algunas ocasiones, información rápida de las complicaciones $^{6}$.

Personal de psicología. Entrena al paciente en estrategias de intervención cognitivo-conductual para el control del dolor. Capacita al paciente en el reconocimiento de las emociones asociadas al dolor y restablece la sensación de autoeficacia ${ }^{3}$. En la figura 1 se observan las líneas de acción del equipo multidisciplinario de la CdDyCP para cada una de las modalidades de atención.

\section{Atención al paciente: Consulta externa}

Durante la consulta, la jefa de servicio y el residente médico valoran la condición actual del paciente, la semiología del dolor y la situación financiera. Todo ello con la finalidad de proporcionar un tratamiento basado en la econofarmacia que garantice un mayor apego al tratamiento. Simultáneamente, el residente de medicina conductual, por medio de una entrevista conductual inicial, evalúa la interacción entre paciente-familiar-personal de salud, la dinámica familiar y las redes de apoyo social. Identifica las principales problemáticas psicológicas en el paciente y cuidadores, así como, el nivel de conocimiento y conciencia del diagnóstico, estilo de vida y pronóstico médico ${ }^{7}$. De ser necesario se realiza intervención en las crisis ante la entrega de malas noticias o ante manifestaciones de labilidad emocional y llanto.

Si el psicólogo detecta una situación problemática, al término de la consulta médica realiza una entrevista clínica complementaria con el fin de evaluar con mayor detalle la conducta problemática. Se puede acompañar con el uso de escalas estandarizadas de tipo unidimensional o multidimensional ${ }^{8,9}$ o medidas estandarizadas de autoinforme y procedimientos observacionales ${ }^{10,11}$. La elección del tratamiento psicológico se determina considerando los criterios diagnósticos del Manual Diagnóstico y Estadístico de los Trastornos Mentales 
TRABAJO MULTIDISCIPLINARIO DE LA CLÍNICA DEL DOLOR Y CUIDADOS PALIATIVOS

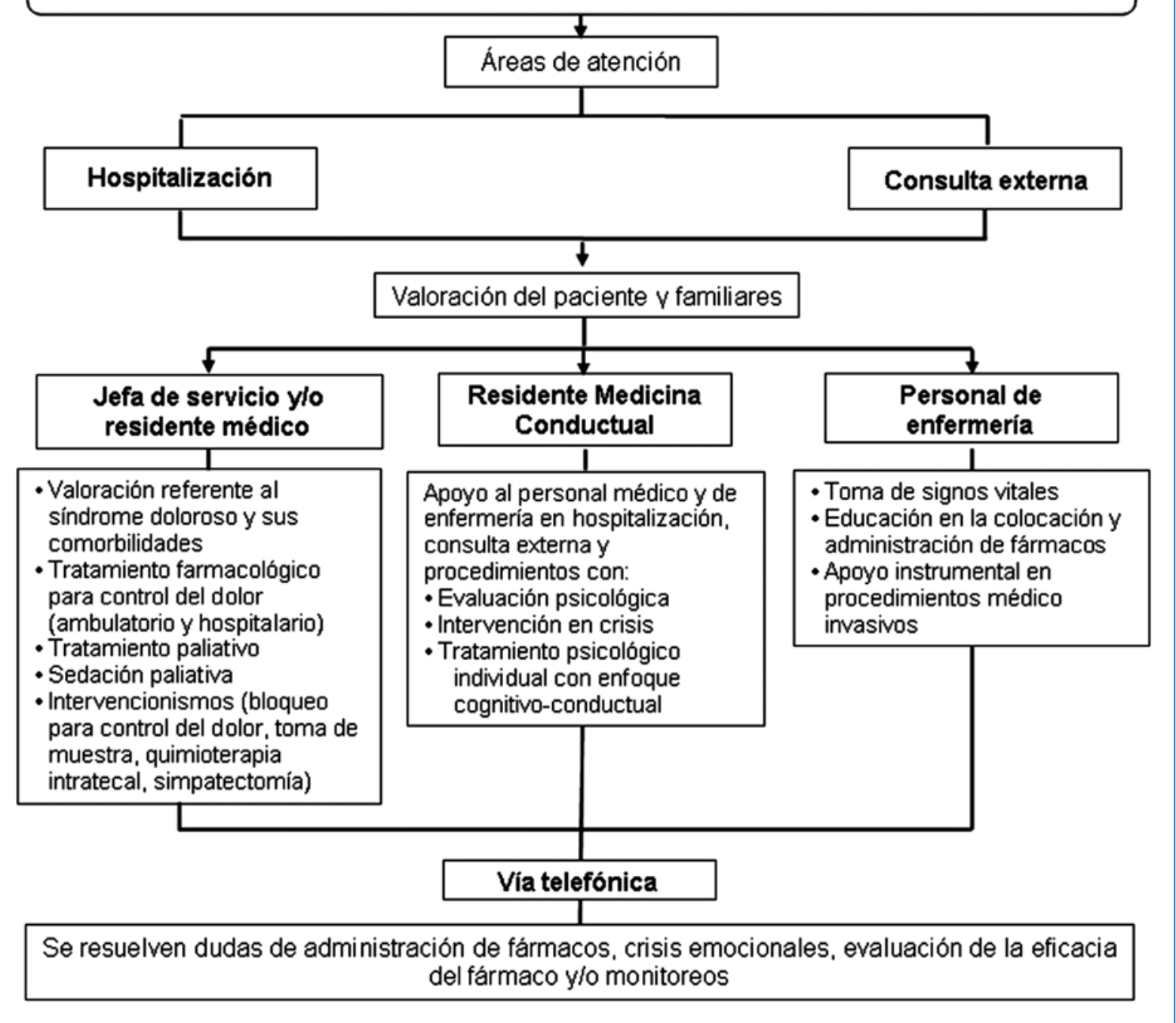

Figura 1. Acciones del equipo multidisciplinario de la Clínica del Dolor y Cuidado Paliativos.

(DSM-5, por sus siglas en inglés) y los déficits y excesos que manifieste el paciente, el cual es atendido de manera subsecuente en la consulta externa por medicina conductual. La línea de acción puede observarse en la figura 2.

Al término de la valoración médica, si el paciente no tiene características de un síndrome doloroso, se deriva a su servicio de base para continuar con el tratamiento sugerido por los médicos; sin embargo, si presenta dolor, seguirá siendo atendido por consulta externa en CdDyCP. En caso de que la farmacología no provea los resultados esperados, se recurre a métodos intervencionistas, por ejemplo, la administración epidural de esteroides, bombas implantables, infiltración de ozono, bloqueos nerviosos y tratamiento con radiofrecuencia ${ }^{12}$. En este tipo de métodos es importante la intervención del médico conductual, ya que promueve una conducta activa en los pacientes con el fin de que este tipo de tratamiento sea tolerable para ellos, además de establecer componentes de relación que faciliten el vínculo de confianza entre médico-paciente ${ }^{13}$. Este tipo de métodos intervencionistas aumentan la percepción de dolor a nivel físico por la extensión de la lesión o la tensión muscular, pero también a nivel emocional, por el incremento del dolor debido a la ansiedad o por procesos cognitivos producidos por el método médico ${ }^{14}$. En ese sentido, el médico conductual puede apoyar con ejercicios para favorecer la relajación, instrucciones 


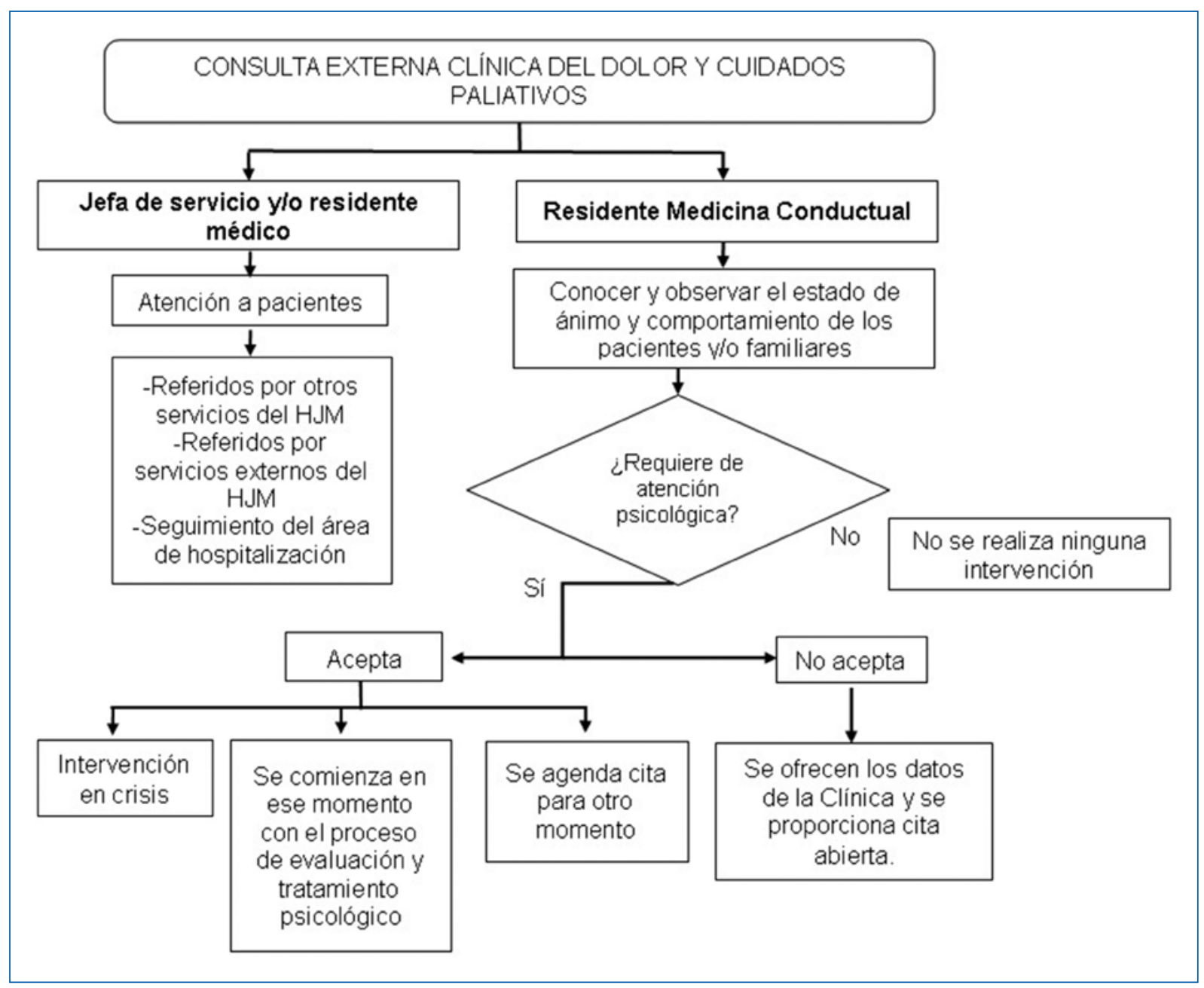

Figura 2. Valoración inicial del paciente en Consulta Externa. HJM: Hospital Juárez de México

autodirigidas (distracción, imaginería, hablarse a sí mismo de manera automotivante), y de esta forma lograr la sensación de control sobre el propio dolor y estrategias de afrontamiento activo ${ }^{15,16}$.

\section{Atención del paciente en cuidados paliativos}

En esta unidad médica pueden ingresar pacientes con enfermedades oncológicas y no oncológicas. Se ofrecen cuidados paliativos, es decir, se ofrece aminorar los síntomas para mejorar la calidad de vida del paciente y disminuir la sobrecarga del cuidador primario. Cuando el paciente llegue a la fase terminal y cumpla con las características que implica la voluntad anticipada, se puede dar como tratamiento la sedación paliativa (disminución deliberada del nivel de conciencia del enfermo mediante la administración de los fármacos apropiados con el objetivo de evitar un sufrimiento intenso causado por uno o más síntomas refractarios), cuyo énfasis está en el seguimiento del paciente durante esta etapa de su vida, sin prolongar ni acortar la vida, respetando el momento natural de la muerte ${ }^{17}$. Ante esta situación, la labor multidisciplinaria es crucial debido a los factores biopsicosociales que se presentan, por una parte, está la atención biomédica a los pacientes (Fig. 3) y, por otra, el apoyo psicosocial a los pacientes y sus familiares. En este proceso de atención, el médico conductual entrena a los involucrados en estrategias de afrontamiento activas, la expresión de emociones y la búsqueda de apoyo social, además se proporciona educación y psicoeducación en conjunto con los médicos y el servicio de enfermería respecto a la función de los medicamentos, la importancia del apego al tratamiento y de los cuidados en casa para el paciente. 


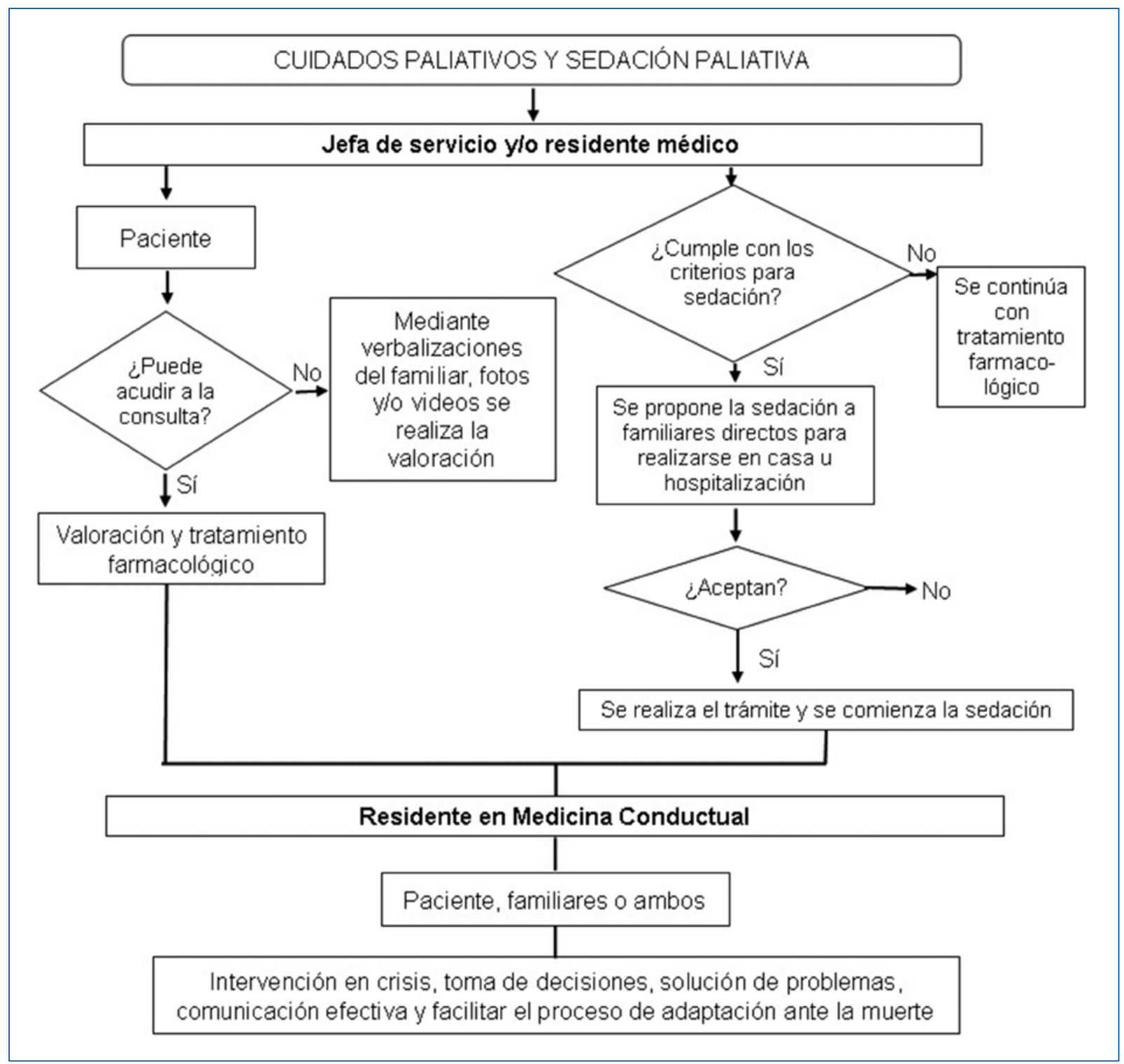

Figura 3. Valoración y tratamiento en Cuidados Paliativos y Sedación Paliativa.

\section{Atención al paciente: Hospitalización}

El equipo multidisciplinario de la CdDyCP (médicos, psicólogo y enfermera) realiza la visita con el paciente, el método de exploración y evaluación es el mismo que se sigue en la consulta externa. Si el paciente amerita ser atendido por un mayor tiempo, será parte del censo y se monitorizará diariamente para corroborar la eficacia del tratamiento médico y psicológico. Durante las interconsultas, el médico conductual realiza la observación clínica de la condición del paciente y del estado de ánimo (en caso de que se presente alguna crisis, se realiza la intervención). Si el paciente va a ser atendido por la $\mathrm{CdDyCP}$, se realiza una entrevista para valorar si requiere de tratamiento psicológico de seguimiento. Si el paciente es dado de alta y es necesario continuar con la intervención psicológica fuera del hospital, se da continuidad por consulta externa de la CdDyCP (Fig. 4).

Al igual que en la consulta externa, la evaluación adecuada incluye no solo conocer la semiología del dolor, sino también el conjunto de creencias y comportamientos manifiestos emitidos por el paciente (creencias relacionadas al dolor y su control, esperanza, toma del medicamento) y sus familiares (la atención que otorgan ante las quejas de dolor del enfermo y el apoyo social proporcionado durante la enfermedad) ${ }^{18}$. Con estos datos, el especialista en medicina conductual 


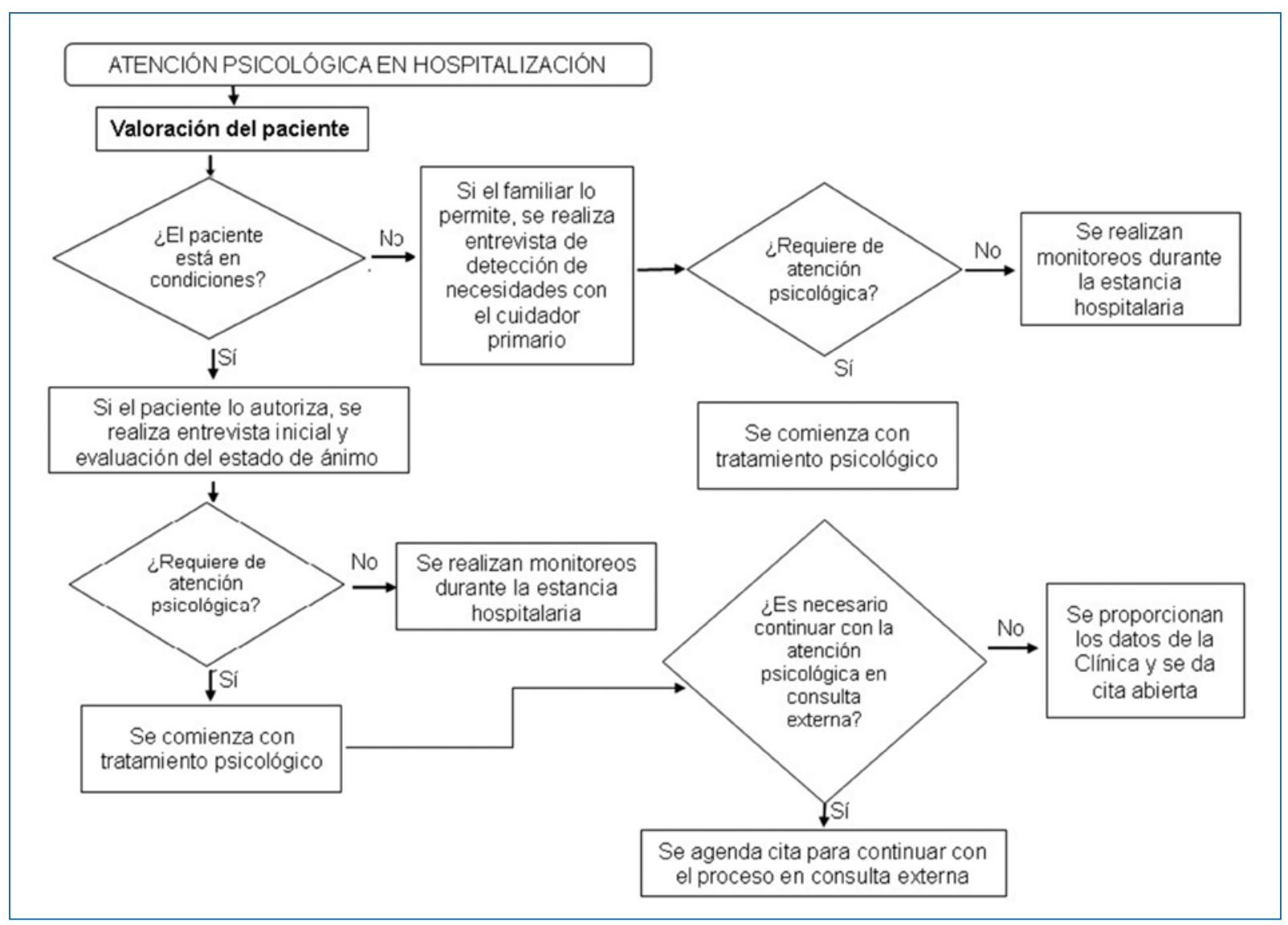

Figura 4. Atención psicológica en hospitalización.

elabora un análisis funcional de la conducta del paciente y con base en este se diseña e implementa un plan de intervención ${ }^{19}$.

Los trastornos psicológicos más frecuentes en pacientes con dolor son: trastornos adaptativos con estado de ánimo depresivo, ansioso y mixto, insomnio, irritabilidad, sentimientos de miedo e indefensión, episodios de angustia, disfunción sexual, problemas familiares, pérdida de roles y aislamiento social ${ }^{20}$. La labor del médico conductual es importante, ya que el dolor puede cronificarse o exacerbarse por factores cognitivos, emociones negativas y estilos de afrontamiento al dolor no funcionales ${ }^{11,21}$.

Por lo tanto, en pacientes con dolor crónico, la meta de la intervención psicológica mediante terapia cognitivo-conductual es entrenar en habilidades que permitan al paciente afrontar de manera adaptativa el dolor, debido a que la discapacidad será mayor o menor en función de cómo se evalúe y afronte el estímulo doloroso ${ }^{22}$. Por ende, esta intervención fomenta una actitud activa frente al dolor, aumenta la autoeficacia, las estrategias dirigidas a la solución de problemas y para la búsqueda de apoyo social, la regulación de emociones mediante el uso de técnicas para la relajación (respiración diafragmática, relajación muscular progresiva e imaginación guiada) y modifica los esquemas cognitivos de los pacientes, por ejemplo, con el uso de psicoeducación ${ }^{3,10,11}$.

\section{Valoración de los cuidadores y familiares de pacientes}

El cuidador primario cotidianamente se enfrenta a situaciones novedosas con su paciente, como consecuencia, puede sufrir pérdidas de control personal y presentar alteraciones en la salud física y emocional ${ }^{23}$. A nivel emocional se pueden manifestar sentimientos tales como tristeza, culpa, rabia, resentimiento y sentimientos de incapacidad ${ }^{24}$, por tanto, es necesario realizar un abordaje psicológico con técnicas dirigidas al control de la ansiedad y/o el estrés, toma de decisiones, mejora de la comunicación médico-paciente, y optimizar la calidad de vida y percepción de apoyo social ${ }^{25}$. 


\section{Atención al personal de salud}

El médico conductual no se limita a atender pacientes y familiares del enfermo, sino también incluye a los profesionales de salud, debido al malestar psicológico que genera trabajar en la CdDyCP. Los programas de intervención basados en la terapia cognitivo-conductual han demostrado ser eficaces, además de que tienen la ventaja de ser estructurados, con amplia evidencia empírica y de corta duración ${ }^{26}$. En una investigación realizada por Gómez, et al., en $2017^{27}$, se encontró que una intervención bajo este enfoque puede modificar algunas respuestas de estrés, ansiedad y depresión en residentes médicos. Concluye, además, que el trabajo oportuno con esta población es importante, ya que este tipo de variables psicológicas repercuten en la calidad de la atención de los pacientes y, por ende, en la reducción de la inversión pública en los servicios de salud.

Atendiendo esta demanda, en la CdDyCP se realizan anualmente y como estrategia educativa para los residentes talleres y clases destinados al control del estrés, ansiedad y respuestas depresivas, así como entrenamiento de habilidades comunicativas y emisión de malas noticias.

\section{Conclusión}

El equipo multidisciplinario de la CdDyCP tiene como meta fomentar la adherencia al tratamiento para el control del dolor y otros síntomas y, así, mejorar la calidad de vida, tanto en el paciente como en el cuidador primario. La colaboración del médico conductual puede favorecer los comportamientos de adherencia por medio de psicoeducación para disminuir dudas acerca del tratamiento y posibles efectos secundarios, además, la intervención cognitivo-conductual permite modificar la percepción que el paciente tiene respecto a su enfermedad, favorece la sensación de control sobre el dolor y, en ese sentido, se puede lograr que se retomen algunas actividades de la vida cotidiana ${ }^{28}$. Evidentemente, las características de los usuarios de la CdDyCP, las condiciones de trabajo en el contexto hospitalario, lidiar con la muerte constantemente y observar el sufrimiento de los pacientes resultan en problemas emocionales en el personal de salud, motivo por el cual el soporte que proporciona el especialista en medicina conductual se vuelve fundamental ${ }^{29}$.

El trabajo sistemático permite el alcance de las metas que tiene hoy por hoy la CdDyCP, sin embargo, se considera necesaria la inclusión de un trabajador social, debido a que podría ayudar a los familiares y/o cuidadores a ampliar sus redes de apoyo, y evitar que la enfermedad del paciente los aleje de sus actividades diarias ${ }^{30}$. El trabajador social es el encargado de informar, orientar y gestionar recursos sociosanitarios para el paciente y, así, favorecer una adecuada inserción social. También evalúan las necesidades del paciente y sus cuidadores para fungir como enlace entre ellos y las instituciones existentes, esto conlleva a una reducción de costos del cuidado de la salud ${ }^{31,32}$.

Como se mencionó, la CdDyCP trabaja multidisciplinariamente, es decir, toma decisiones basadas en la suma de las competencias de las distintas áreas de atención profesional, pero se mantienen dentro de los límites de sus áreas, como resultado se obtienen metas distintas para cada profesión, usando metodologías separadas, por lo tanto, es la suma mas no la integración de disciplinas $^{33,34}$. Dicho esto, se propone que la CdDyCP trabaje bajo un enfoque interdisciplinario en el que se integren las distintas áreas de atención, y aporten sus problemas, conceptos y métodos de investigación, cuyas metas sean compartidas y tengan funciones comunes ${ }^{33,35,36}$.

\section{Conflicto de intereses}

Los autores declaran no tener conflicto de intereses alguno.

\section{Financiamiento}

Los autores no recibieron patrocinio para llevar a cabo este artículo.

\section{Responsabilidades éticas}

Protección de personas y animales. Los autores declaran que los procedimientos seguidos se conformaron a las normas éticas del comité de experimentación humana responsable y de acuerdo con la Asociación Médica Mundial y la Declaración de Helsinki.

Confidencialidad de los datos. Los autores declaran que en este artículo no aparecen datos de pacientes.

Derecho a la privacidad y consentimiento informado. Los autores declaran que en este artículo no aparecen datos de pacientes.

\section{Bibliografía}

\footnotetext{
1. IASP. Dolor, diagnóstico y tratamiento. PAIN. 2009;137:473-7.

2. Guajardo J, Plancarte R. Manejo intervencionista del dolor crónico. Revista Digital Universitaria. 2006;7(4):2-6
} 
3. Velázquez N, Vázquez L, Alvarado S. Ansiedad Asociada al Dolor Agudo en Pacientes Oncológicos. Cancerología. 2009;4:19-29.

4. Hospital Juárez de México (HJM). Manual de organización específico del servicio de enfermería de Clínica del Dolor. 2014.

5. Academia Nacional de Medicina (ANMM). La clínica del dolor. Boletín de Información Clínica Terapéutica de la ANMM 2014;57(3):53-6. Disponible en: http://www.scielo.org.mx/pdf/facmed/v57n3/2448-4865-facmed57-03-00053.pdf

6. Montealegre, D. Retos para enfermería en el cuidado de personas con dolor: una forma de humanización. RevCuid. 2014;5(1):679-88.

7. De la Fuente R. La enseñanza de la psicología médica en la Facultad de Medicina de la UNAM. RevFacMed UNAM. 2006;49(2):51-3.

8. Cid J, Acuña J, de Andrés J, Díaz L, Gómez-Caro L. ¿Qué y cómo evaluar al paciente con dolor crónico? Evaluación del paciente con dolor crónico. Rev Med Clin Condes. 2014;25(4):687-97.

9. Bragard D, Decruynaere C. Evaluación del dolor: aspectos metodológicos y uso clínico. EMC-Kinesiterapia - Medicina Física. 2010;31(4):1-11

10. Fernández-Ballesteros R. Los autoinformes. En: Fernández-Ballesteros, $R$ (Dir.). Evaluación psicológica. Conceptos, métodos y estudio de casos.

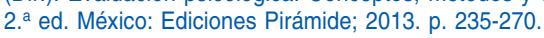

11. Turk D, Melzack R. Handbook of pain assessment, $2^{\text {nd }}$ ed. New York: Guilford Press; 2012.

12. Van Hecke O, Torrance N, Smith B. Chronic pain epidemiology and its clinical relevance. Brit J Anaesth. 2013;111(1):13-18

13. Navarro M, Silveria P. ¿Por qué una psicología médica? Salud Militar. 2005;27(1):83-90

14. Melzack R, Wall P. Pain mechanism: a new theory. Science. 1965;150:971-9.

15. Almendro $\mathrm{M}$, Clariana $\mathrm{S}$, Hernández $\mathrm{S}$, Rodríguez $\mathrm{C}$, Camarero $\mathrm{B}$, del Río T. Programa de atención psicológica al dolor crónico: El reto de un afrontamiento activo. Rev Asoc Esp Neuropsiq. 2010;31(110):213-27.

16. Ruvalcaba G, Domínguez B. La terapia psicológica del dolor crónico. Psicología y Salud. 2009;19(2):247-52.

17. Organización Médica Colegial (OMC) y Sociedad Española de Cuidados Paliativos (SECPAL). Guía de Sedación Paliativa. Madrid; 2011.

18. Turner J, Chapman R. Psychological Interventions for Chronic Pain: a Critical Review. I. Relaxation Training and Biofeedback. Pain. 1982;12:1-12.

19. Bravo C, Mora-Miranda M. Análisis Funcional y diseño de intervención en el ámbito hospitalario. En: Reynoso L, Becerra A (Coords.). Medicina Conductual: Teoría y Práctica. 1. ${ }^{\text {a }}$ ed. México: UNAM Qartuppi, S. de R.L. de C.V; 2014. p. 101-128.

20. Esteve R, Ramírez C, López A. Aspectos emocionales en el dolor. Actualizaciones Dolor. 2001;2(4):252-61.

21. Araos B. Manejo multidisciplinario del Dolor Crónico. Rev Med Clin Condes. 2007;18(3):222-8.
22. Lazarus R, Folkman S. Estrés y procesos cognitivos. Barcelona: Ediciones Martínez Roca; 1986.

23. Ribas J, Castel A, Escalada B, Ugas L, Grau C, Magarolas R, et al. Trastornos psicopatológicos del cuidador principal no profesional de pacientes ancianos. Rev Psiquiatría Fac Med Barna. 2000;27(3):131-4.

24. Rabow M, Hauser J, Adams J. Supporting family caregivers at the end of life: "they don't know what they don't know". JAMA. 2004:291(4):483-91.

25. Zabalegui A, Canalias M, Fornas C, Robles D. Cuidando al cuidador. Guía práctica y recomendaciones para el cuidador. $1 .^{\mathrm{a}}$ ed. Barcelona: Hospital Clínic de Barcelona; 2012. Disponible en: https://portal.hospitalclinic.org/uploads/media/default/0001/02/3bb8f30560548ecfc20f15db38ac0e0da8e14a01.pdf

26. Fernández-Alcántara M, Ortega-Valdivieso A, Pérez-Marfil M, García-Caro M, Cruz-Quintana F. Funciones y situación actual de la intervención de los psicólogos en Cuidados Paliativos. Psicooncología. 2014;11(1):163-72.

27. Gómez A. Intervención Cognitivo-Conductual para el control de estrés, ansiedad y depresión en residentes médicos (Tesis de maestría). Universidad Nacional Autónoma de México; 2017.

28. Benito G, Nadador V, Fernández-Alcnatud J, Hernández-Salvan J, Ruiz-Castro M, Riquelme I. Intervenciones del psicólogo en las Clínicas del Dolor: Una propuesta desde la experiencia de la Unidad del Dolor del Hospital Universitario Príncipe de Asturias en Alcalá de Henares, Madrid. Rev Soc Esp Dolor. 2006;13(4):254-62.

29. Ortega C, López F. Intervención psicológica en cuidados paliativos: revisión y perspectivas. Clínica y Salud. 2005;16(2):143-60.

30. IASP. IASP Curriculum Outline for Pain in Social Work. 2018. Disponible en: http://www.iasp-pain.org/Education/CurriculumDetail.aspx?ItemNumber $=4956$

31. Parker D, Wittenberg-Lyles E, Washington K, Sehrawat S. Social Work Role in Pain Management with Hospice Caregivers: A National Survey. $J$ Soc Work End Life Palliat Care. 2009;5(1-2):61.

32. Bermejo JC, Díaz-Albo E, Sánchez E. Manual Básico para la Atención Integral en Cuidados Paliativos. Madrid: Cáritas; 2011.

33. Choi B, Pak A. Multidisciplinarity, interdisciplinarity and transdisciplinarity in health research, services, education and policy: 1. Definitions, objectives, and evidence of effectiveness. Clin Investig Med. 2006; 29(6):351-64

34. Giusti E, Castelnuovo G, Molinari E. Differences in Multidisciplinary and Interdisciplinary Treatment Programs for Fibromyalgia: A Mapping Review. Pain Res Manag. 2017;2017:7261468.

35. Pérez N, Setién E. La interdisciplinariedad y la transdisciplinariedad en las ciencias: una mirada a la teoría bibliológico-informativa. Acimed. 2008;18(4). Disponible en: http://scielo.sld.cu/pdf/aci/v18n4/aci31008.pdf

36. Gonzalez E. La salud pública como campo transdisciplinar. Revista Facultad Nacional de Salud Pública. 2007:25(1):71-7. 